\title{
Blocking L-type Voltage-Gated Calcium Ion Channels Changes the Intensity of Protein Synthesis in Metanephric Cells
}

\author{
Yelena Yu. Shapovalova, PhD, ScD*; Liliana A. Kutuzova, PhD; \\ Svetlana V. Kharchenko, PhD; Svetlana A. Vasilenko \\ Medical Academy named after S.I. Georgievsky of Vernadsky CFU, Simferopol, Russia
}

\begin{abstract}
The purpose of this research was to study the effect of L-type voltage-gated calcium channels (L-VGCC) blocking on mRNA content and intensity of protein synthesis in cells of the metanephros.

Materials and Methods: The study was performed on 27 outbred Wistar rats weighing 250-270 g from 5 to 7 months of age. Pregnant females were divided into 3 groups of 9 individuals each. Group1 (the control group) included intact pregnant females treated with distilled water; Group 2 (the first experimental group) included animals receiving a therapeutic dose of nifedipine; Group 3 (the second experimental group) included animals receiving a toxic dose of nifedipine. The dihydropyridine nifedipine (SigmaAldrich, Gillingham, UK) was used as a $\mathrm{Ca} 2+$ channel blocker. Nifedipine was dissolved in distilled water. The dose of nifedipine was calculated based on therapeutic and toxic doses for humans. The therapeutic dose for rats was $127 \mathrm{mg} / \mathrm{kg}$, toxic dose $-762 \mathrm{mg} /$ $\mathrm{kg}$. In each group, when rats reached Day 14, 15, 16 and consistently up to 22 of gestation, they were removed from the experiment by decapitation under ether anesthesia. The embryos and fetuses were removed, subjected to external examination and quickly fixed with $10 \%$ neutral formalin entirely up to the age of 15 days. At an older age, both metanephros were removed from the fetuses by preparation and fixed with $10 \%$ neutral formalin. The intensity of protein synthesis was estimated by the content of mRNA in the cytoplasm.

Results: The development of the metanephros in rats of the control group was accompanied by a gradual decrease in the content of mRNA, indicating the activity of protein synthesis in the cell cytoplasm of epithelial and mesenchymal buds of the metanephros. In the fetuses of rats whose mothers received therapeutic and toxic doses of nifedipine, statistically significant changes in the content of mRNA in the cytoplasm of the metanephric cells were observed. These changes were most significant in the metanephric cells after administration of the toxic dose. On ED 17, an L-VGCC blockade with a therapeutic dose of nifedipine led to a paradoxical reaction of cells with an increase in protein synthesis, compared with the control group.

Conclusion: Both doses of nifedipine have a greater effect on the content of mRNA in the cytoplasm of epithelial cells, compared to mesenchymal buds. (International Journal of Biomedicine. 2019;9(2):150-154.)
\end{abstract}

Key Words: metanephros $\bullet$ voltage-gated calcium channels $\bullet$ nifedipine $\bullet$ protein synthesis

\section{Introduction}

Calcium ions play an important role in regulation of many vital physiological functions and metabolic processes. By penetrating the cell, they activate bioenergetic processes, as the transformation of ATP into cyclic AMP and protein phosphorylation. ${ }^{(1)}$ Physiological activity is characteristic only of ionized calcium $\left(\mathrm{Ca}^{2+}\right)$ - unbound calcium. $\mathrm{Ca}^{2+}$ ions are unique in that they not only carry a charge but they are also the

*Corresponding author: Prof. Elena Shapovalova, PhD, $S c D$. Head of the Department of Histology \& Embryology of the Medical Academy named after S.I. Georgievsky of Vernadsky CFU. Simferopol, Russia.E-mail: Shapovalova_L@mail.ru most widely used of diffusible second messengers. Voltagegated calcium channels (VGCCs) are the primary mediators of depolarization-induced calcium entry into the cell.(2)

Based on pharmacological and biophysical properties, VGCCs are classified into 3 families: the L-type, the $\mathrm{P} / \mathrm{Q} / \mathrm{R} /$ $\mathrm{N}$-type and the T-type channels. ${ }^{(2)}$ The long-lasting (L-type) VGCCs (L-VGCCs) have been shown to play crucial roles in $\mathrm{Ca}^{2+}$ homeostasis of excitable cells. L-VGCCs are present in the heart, smooth and skeletal muscle, and some neurons. The importance of L-VGCCs is demonstrated by the clinical efficacy of $\mathrm{Ca}_{2}{ }^{+}$channel blockers in certain disease conditions.

A number of postnatal kidney diseases are known to be associated with abnormalities during prenatal organogenesis. Fetal development occurs in a hypercalcemic environment 
compared with the postnatal period. ${ }^{(3)}$ The few experimental studies found in the world's scientific bases suggest that calcium activity contributes to many aspects of kidney development and its decline leads to the formation of associated diseases..$^{(4)}$ Very few studies have been devoted to the effect of blocking the passage of calcium ions into the cell through L-VGCCs on the development of the metanephros and the associated protein synthesis in its cells.

The purpose of this research was to study the effect of L-VGCC blocking on mRNA content and intensity of protein synthesis in cells of the metanephros.

\section{Materials and Methods}

\section{Design of the experiment}

The study was performed on 27 outbred Wistar rats weighing $250-270 \mathrm{~g}$ from 5 to 7 months of age. The animals were housed in keeping with the rules for good laboratory practice (GLP). The experiments were performed in accordance with the norms for the humane treatment of animals, which are regulated by Directive 86/609/EEC on the protection of animals used for experimental and other scientific purpose .Dated (timed) gestational age was determined using a daily study of vaginal smears. Before the start of the experiment, the estrous cycle was investigated in all females. Animals with its disturbances were excluded from the experiment. The female rats included in the experiment, in the cycle stage corresponding to the late proestrus or early estrus, were placed together with male rats during the night, and in the morning of the next day, vaginal smears were taken from females. The fact of pregnancy was established by the presence of sperm in the vaginal secretion, and the day of its discovery was considered the first day of pregnancy. Pregnant females were divided into 3 groups of 9 individuals each.

Group1 (the control group) included intact pregnant females treated with distilled water; Group 2 (the first experimental group) included animals receiving a therapeutic dose of nifedipine; Group 3 (the second experimental group) included animals receiving a toxic dose of nifedipine. The dihydropyridine nifedipine (Sigma-Aldrich, Gillingham, UK) was used as a $\mathrm{Ca} 2+$ channel blocker. Nifedipine was dissolved in distilled water. The dose of nifedipine was calculated based on therapeutic and toxic doses for humans. The therapeutic dose for rats was $127 \mathrm{mg} / \mathrm{kg}$, toxic dose $-762 \mathrm{mg} / \mathrm{kg}$.

Nifedipine or distilled water was administered from the eighth day of pregnancy once, at the same time of day, intragastrically using a probe. During the experiment, the condition and behavior of the animals were monitored with mandatory weighing once a week to identify the toxic effects of the drug. In each group, when rats reached Day 14, 15, 16 and consistently up to 22 of gestation, they were removed from the experiment by decapitation under ether anesthesia. Given the presence of circadian rhythms, decapitation was performed at the same time of day. At the opening of the abdominal cavity and the horns of the uterus, the embryos and fetuses were removed, subjected to external examination and quickly fixed with $10 \%$ neutral formalin entirely up to the age of 15 days. At an older age, both metanephros were removed from the fetuses by preparation and fixed with $10 \%$ neutral formalin. Morphological examination of metanephros

Serial paraffin sections $5 \mu \mathrm{m}$ thick were made from the obtained material. Tissue sections were stained with H\&E. The intensity of protein synthesis was estimated by the content of mRNA in the cytoplasm. ${ }^{(6)}$ The researchers in our $1 a b^{(7)}$ found that cells of embryonic tissues synthesize protein intensively, both for plastic and metabolic purposes. mRNA in the cytoplasm of cells was determined by the method of Einarson using gallocyanin-chromic alum. ${ }^{(8)}$ The intensity of cytoplasmic staining was assessed using the computer program Aperio Image Scope 2008.

Statistical analysis was performed using the statistical software «Statistica» (v6.0, StatSoft, USA) and Microsoft Excel 2007. Baseline characteristics were summarized as frequencies and percentages for categorical variables and as mean \pm SEM for continuous variables. The Mann-Whitney (U Test) was used to compare the differences between the two independent groups. The Wilcoxon criterion was used to compare the differences between the paired samples. A probability value of $P<0.05$ was considered statistically significant.

\section{Results}

In Group 1, the metanephros in rat embryos was determined by embryonic day (ED) 14. On ED 15, a metanephrogenic zone was formed along the periphery of the bud, in which at this time and on the subsequent ED 16, primary renal tubules were formed, indicating the beginning differentiation of nephrons. Mesenchyme, which lies in the area of the future medulla, was represented by loose tissue. At the stage of prenatal embryo development, corresponding to 16 days, Einarson stains were intensively staining the cyan-blue cytoplasm of the cells of the primary renal tubules and the surrounding mesenchyme, indicating a high content of mRNA in them. On ED 17, the epithelial and mesenchymal buds of the metanephros rapidly underwent further changes. The metanephros contained four generations of the branches of the metanephritic diverticulum, and an active neoplasm of the nephron primordia occurred. Their number per unit area was much larger than in the previous stage. This process of differentiation was accompanied by a decrease in the amount of mRNA in the cytoplasm of mesenchymal cells by $41.2 \%$, in the tubule epithelium - by $18.8 \%$ compared with the same structures of the metanephros on ED 16.

By ED 19, the metanephros continued to grow, surrounded by a pronounced capsule. The cortex and medulla were well defined. In the cortical substance, a small amount of the metanephric tissue was maintained, consisting of densely adjacent cells with round nuclei and basophilic cytoplasm. In the subcapsular zone, the rudiments of the fourth generation nephrons were located, which were transformed into S-shaped structures, the most immature tabs of the nephrons. As we approached the medulla, more and more differentiated nephrons were generated. The pelvis with a rather wide cavity was lined with transitional epithelium, and epithelial folds were found. The transitional epithelium also appeared in large 
and small calyces. In the metanephros on ED 19, there was a weakening of the color, according to Einarson, concerning all the structures studied. In the cytoplasm of mesenchymal cells, the weakening was $61.1 \%$, and in the tubular epithelium it was $34.7 \%$, compared with the same structures of the metanephros on ED 17.

In the metanephros on ED 21-22, the renal structures tightly adhered to each other. The buds of the nephrons did not form, but the existing buds grew and differentiated. Four generations of nephrons were detected. Fourth generation immature nephrons occupied a subcapsular position. The nephrons of the first 3 generations were much more differentiated. Tubular differentiation continued. Thin tubules were lengthened and penetrated deeper into the medulla. Collecting tubules were dilated, covered with prismatic or cubic epithelial cells. In the metanephros, clear demarcation between cortex and medulla was observed. An allocation of renal pyramids and pillars continued. In the pelvis, large and small calyces were lined with transitional epithelium, and we detected folding of the epithelial lining. The kidney was surrounded on the outside by a well-formed connective tissue capsule. In the fetus of this age, the RNA content in the cells of the kidney buds continued to decline. The most pronounced decrease was found in the cytoplasm of mesenchymal cells, where it reached $74.3 \%$, compared with ED 19. A decrease in the RNA content was not so active in the epithelium of the tubules and was 37.4\%.

In Group 2, the changes in the mRNA content were already detected on ED 17. Its amount in epithelial cells increased significantly compared to Group 1 (Table 1) and more significantly than in mesenchymal cells (Table 2).

Table 1.

Dynamics of the mRNA content in the cytoplasm of epithelial cells of rat metanephros in study groups

\begin{tabular}{|l|c|c|c|}
\hline \multicolumn{1}{|c|}{ Group } & ED 17 & ED 19 & ED 21 \\
\hline Group 1 & $100 \%$ & $100 \%$ & $100 \%$ \\
\hline Group 2 & $+10.79 \%$ & $+13.86 \%$ & $-26.60 \%$ \\
\hline Group 3 & $-27.58 \%$ & $-38.89 \%$ & $-88.40 \%$ \\
\hline
\end{tabular}

Table 2.

Dynamics of the mRNA content in the cytoplasm of mesenchymal cells of rat metanephros in study groups

\begin{tabular}{|l|c|c|c|}
\hline \multicolumn{1}{|c|}{ Group } & ED 17 & ED 19 & ED 21 \\
\hline Group 1 & $100 \%$ & $100 \%$ & $100 \%$ \\
\hline Group 2 & $+8.19 \%$ & $-0.72 \%$ & $-6.80 \%$ \\
\hline Group 3 & $-22.12 \%$ & $-31.7 \%$ & $-72.10 \%$ \\
\hline
\end{tabular}

On ED 19, the positive dynamics of the RNA content in the cytoplasm of the bud cells of the metanephros was traced only for the epithelial cells of the tubules, while in the cells of mesenchymal buds, this content began to fall, although not statistically significantly. The most pronounced changes in the developing rat metanefrosis after the introduction of a therapeutic dose of nifedipine appeared on ED 21, when the RNA content in epithelial cells and mesenchyme decreased.

In Group 3, the changes in the mRNA content in the cytoplasm of metanephros cells were more pronounced. Significant shifts in the direction of the mRNA content inhibition began from ED 17 and by ED 21 reached peak values (Tables 1 and 2). Both doses of nifedipine had a greater effect on the mRNA content in the cytoplasm of epithelial cells than they did in cells of mesenchymal buds.

\section{Discussion}

In the literature, there is evidence that calcium signaling is necessary for regulating the processes of differentiation of the metanephric tubules. ${ }^{(9)}$ It was also found that calcium activity contributes to multiple aspects of kidney development and associated diseases. ${ }^{(10)}$ In recent years, several studies have pinpointed an unsuspected role of calcium in determining the pronephric territory and for converting metanephric mesenchyme into nephrons. Influx of calcium and calcium transients has been recorded in the pool of renal progenitors to allow tubule formation, highlighting the occurrence of calcium-dependent signaling events during early kidney development. ${ }^{(11)}$ However, the presence of calcium channels directly in the metanephric tubules has not yet been described. They are found only in the smooth muscles of the kidney vessels. ${ }^{(12)}$

Studies over the last several decades have revealed that vascular smooth muscle cells (VSMs) express a variety of $\mathrm{Ca}^{2+}$-permeable channels that coordinate a regulation of intracellular $\mathrm{Ca}^{2+}$ concentration $\left(\left[\mathrm{Ca}^{2+}\right] \mathrm{i}\right)$. Changes in $\left[\mathrm{Ca}^{2+}\right] \mathrm{i}$ are produced by $\mathrm{Ca}^{2+}$ influx through voltage-dependent and -independent plasmalemmal $\mathrm{Ca}^{2+}$-permeable channels, as well as $\mathrm{Ca}^{2+}$ release from intracellular stores. $\mathrm{Ca}^{2+}$ influx through $\mathrm{L}-\mathrm{VGCCs}$ is the principal mediator of myogenic response. $\mathrm{Ca}^{2+}$ release from intracellular stores through ryanodine receptors (RyRs) and inositol-1,4,5,-trisphosphate receptors (IP3Rs) in the sarcoplasmic reticulum (SR) is an important contributor to $\left[\mathrm{Ca}^{2+}\right] \mathrm{i}$ and VSM excitability. ${ }^{(13)}$

Depending on the physiological stimuli, $\mathrm{Ca}^{2+}$ release from RyRs can cause global elevation of $\left[\mathrm{Ca}^{2+}\right] \mathrm{i}$ to activate actin-myosin interaction for vasoconstriction, and generate local $\mathrm{Ca}^{2+}$ signals in the sarcoplasmic reticulum - plasma membrane junctions to stimulate $\mathrm{Ca}^{2+}$-activated channels to modulate membrane potential and $\mathrm{Ca}^{2+}$ influx via VGCCs. ${ }^{(14)}$

Specific blockade of L-type calcium channels using nifedipine decreases the myoplasmic calcium ion concentration, thus preventing the development of changes in the expression of myosin heavy chains (MHC) and sarcoplasmic/endoplasmic reticulum calcium ATPase (SERCA) isoforms. ${ }^{(15)}$

In a series of theoretical studies, it was proposed that morphogenesis in kidney, lung, and mammary glands can be described as a self-organized process ${ }^{(16)}$ with stochastic components that are modified and controlled by signaling molecules. ${ }^{(17,18)}$ Calcium signals play a central role in these processes. Several studies of the embryonic kidney 
demonstrated that genetically controlled and programmed events play a role in proper branching morphogenesis. ${ }^{(19)}$

JM Kim et al. ${ }^{(20)}$ found spatially expressed L-VGCCs in the peripheral layers of developing epithelial buds and identified the VDCCs as a core signaling mediator for patterning branching architecture. JM. Fontana et al. ${ }^{(17)}$ found that down-regulation of the calcium activity, both by blocking the sarco-endoplasmic reticulum $\mathrm{Ca}^{2+}$-ATPase and by chelating cytosolic calcium, resulted in retardation of branching morphogenesis and a reduced formation of primitive nephrons but had no effect on cell proliferation. Authors proposed that the spontaneous calcium signals observed in metanephric mesenchyme cells are, along with stochastic components, among the factors that contribute to the process that drives branching morphogenesis.

Cells of embryonic tissues intensively synthesize protein, both for plastic and metabolic purposes..$^{(7)}$ The content of mRNA in the cytoplasm reflects the intensity of proteinsynthetic processes in the cell. Protein synthesis is most active in the early stages of organ development, when the morphogenesis of organs is accompanied by the migration, proliferation and differentiation of cells that require a large number of protein molecules. ${ }^{(21)}$ This fact is confirmed by the results of our research. The maximum high mRNA content, estimated by intensity of cytoplasmic staining, in the epithelial and mesenchymal buds of the metanephros was found in the earliest period (ED16) of embryogenesis. With an increase in the gestation period, the color intensity of the cytoplasm of epithelial cells and cells of mesenchymal buds gradually decreased, reaching the lowest values on ED 21. With the introduction of nifedipine in a therapeutic dose (a moderate channel blockade), the content of mRNA in the cytoplasm of the cells of the metanephric tubules, indicating the intensity of protein synthesis, depends on the duration of the L-VGCC blockade. On ED 17, a paradoxical reaction of the cells was recorded with an increase in protein synthesis, compared with the control group. Subsequently, on ED 19 and 21, the content of mRNA in the cytoplasm of epithelial cells and mesenchymal derivatives decreased steadily. With a complete L-VGCC blockade (toxic dose of nifedipine), an inhibition in protein synthesis in the epithelial and mesenchymal buds of the metanephros statistically reliably increased in all studied periods relative to the control group.

\section{Conclusion}

Thus, the development of the metanephros in rats of the control group was accompanied by a gradual decrease in the content of mRNA, indicating the activity of protein synthesis in the cell cytoplasm of epithelial and mesenchymal buds of the metanephros. In the fetuses of rats whose mothers received therapeutic and toxic doses of nifedipine, statistically significant changes in the content of mRNA in the cytoplasm of the metanephric cells were observed. These changes were most significant in the metanephric cells after administration of the toxic dose. On ED 17, an L-VGCC blockade with a therapeutic dose of nifedipine led to a paradoxical reaction of cells with an increase in protein synthesis, compared with the control group. Both doses of nifedipine have a greater effect on the content of mRNA in the cytoplasm of epithelial cells, compared to mesenchymal buds.

\section{Competing Interests}

The authors declare that they have no competing interests.

\section{References}

1. KramerI.IntracellularCalcium.In:Signal Transduction. Third Edition. Elsevier; Amsterdam, The Netherlands; 2016:381-439.

2. Catterall WA. Voltage-gated calcium channels. Cold Spring Harb Perspect Biol. 2011;3(8):a003947. doi: 10.1101/ cshperspect.a003947.

3. Finney BA, del Moral PM, Wilkinson WJ, Cayzac $\mathrm{S}$, Cole $\mathrm{M}$, et al. Regulation of mouse lung development by the extracellular calcium-sensing receptor, CaR. J Physiol. 2008;586:6007-6019.

4. Tena JJ, Neto A, de la Calle-Mustienes E, Bras-Pereira C, Casares F, Gómez-Skarmeta JL. Odd-Skipped Genes Encode Repressors That Control Kidney Development. Dev. Biol. 2007;301:518-531. doi: 10.1016/j.ydbio.2006.08.063.

5. Compendium 2006 - Medicinal Products. In Kovalenko VN, Viktorov AP, editors. K.: Morion; 2006. [In Russian].

6. Spirin AS. [Biosynthesis of proteins, the world of RNA and the origin of life]. Herald of the Russian Academy of Sciences. 2001;71(4):320-328. [Article in Russian].

7. Kharchenko SV. [Changes in the activity of protein synthesis in embryogenesis of the lungs and kidneys of rats after the administration of paracetamol and nimesulide to pregnant females]. World of Medicine and Morphology. 2009;5(3-2):185-188. [Article in Russian].

8. Semchenko VV, Barashkova SA, Nozdrin VI. Histological technique. Omsk, 2006. [In Russian].

9. Leclerc C, Webb SE, Miller A, Moreau M. An increase in intracellular $\mathrm{Ca}^{2+}$ is involved in the differentiation of prenodic tubules in amphibians of Xenopus laevis. Dev Biol. 2008;321(2):357-67. doi: 10.1016/j.ydbio.2008.06.029.

10. Paudel S, Sindelar R, Saha. Calcium Signaling in Vertebrate Development and Its Role in Disease. Int J Mol Sci. 2018;19(11):3390 doi: 10.3390/ijms19113390

11. Gilbert T, Leclerc C, Moreau M. Control of kidney development by calcium ions. Biochimie. 2011;93(12):212631. doi: 10.1016/j.biochi.2011.07.007.

12. Hughes AD. Calcium channels in vascular smooth muscle cells. J Vasc Res. 1995;32:353-370.

13. Ghosh D, Syed AU, Prada MP, Nystoriak MA, Santana LF, Nieves-Cintrón M, Navedo MF. Calcium Channels in Vascular Smooth Muscle. Adv Pharmacol. 2017;78:49-87. doi: 10.1016/bs.apha.2016.08.002.

14. Lin AH, Sun H, Paudel O, Lin MJ, Sham JS. Conformation of ryanodine receptor-2 gates store-operated calcium entry in rat pulmonary arterial myocytes. Cardiovasc Res. 2016;111(1):94-104. doi: 10.1093/cvr/cvw067.

15. Mukhina AM, Altaeva EG, Nemirovskaya TL, Shenkman BS. The role of L-type calcium channels in the accumulation of $\mathrm{Ca} 2+$ in soleus muscle fibers in the rat and changes in the ratio of myosin and serca isoforms in conditions of gravitational unloading. Neurosci Behav Physiol. 2008;38(2):181-8. doi: 10.1007/s11055-008-0027-X. 
16. $\mathrm{Xu} \mathrm{H}$, Sun $\mathrm{M}$, Zhao X. Turing mechanism underlying a branching model for lung morphogenesis. PLoS One. 2017;12(4):e0174946. doi: 10.1371/journal.pone.0174946.

17. Fontana JM, Khodus GR, Unnersjö-Jess D, Blom H, Aperia A, Brismar H. Spontaneous calcium activity in metanephric mesenchymal cells regulates branching morphogenesis in the embryonic kidney. FASEB J. 2019;33(3):4089-4096. doi: 10.1096/fj.201802054R.

18. Blake J, Rosenblum ND. Renal branching morphogenesis: morphogenetic and signaling mechanisms. Semin Cell Dev Biol. 2014;36:2-12. doi: 10.1016/j.semcdb.2014.07.011.
19. Hannezo E, Scheele CLGJ, Moad M, Drogo N, Heer R, Sampogna RV, van Rheenen J, Simons BD. A Unifying Theory of Branching Morphogenesis. Cell. 2017;171(1):242-255.e27. doi: 10.1016/j.cell.2017.08.026.

20. Kim JM, Choi S, Lee SW, Park K. Voltage-dependent Ca2+ channels promote branching morphogenesis of salivary glands by patterning differential growth. Sci Rep. 2018;8(1):7566. doi: 10.1038/s41598-018-25957-w.

21. Vetter MR, Gibley CW. Morphogenesis and histochemistry of the developing mouse kidney. Journal of Morphology. 2005;120(2):135-155. 